\title{
Los inicios de la psicotécnica en Checoslovaquia*
}

\author{
Jiř́ Hoskovec \& Josef Brožek ${ }^{\dagger}$, Universidad Carlos de Praga (República Checa) \\ Recibido: 22 de mayo del 2009 / Aprobado: 9 de julio del 2009
}

Los autores presentan el desarrollo de la psicotécnica en Checoslovaquia, recurriendo a material de archivo. Se proporciona información acerca de los contactos internacionales del Instituto Psicotécnico (Psychotechnicky ústav), así como de las constantes visitas de sus miembros a instituciones extranjeras y viceversa, de figuras destacadas de la psicotécnica internacional a Checoslovaquia (por ejemplo, Meyerson de Francia, Blumenfeld de Alemania, Walther de Suiza). Se comenta asimismo la correspondencia conservada en los archivos entre figuras de la psicotécnica checoslovaca y sus pares en otros países, la recepción de la literatura psicotécnica internacional y la VIII Conferencia Internacional de Psicotécnica celebrada en Praga en 1935.

psicotécnica / Checoslovaquia

\section{The beginnings of psychotechniques in Czechoslovakia}

The authors present the history and development of psychotechniques in Czechoslovakia, using archive material. Sources include the international contacts of the Psychotechnique Institute (Psychotechnicky ústav), the visits of members of the Institute to foreign institutions and the visit of renowned international psychotechnicians, such as Meyerson from France, Blumenfeld from Germany, and Walther from Switzerland. It is also discussed the correspondence stored in archives between some figures of Czechoslovakian psychotechnique and their peers in other countries, the reception of international psychotechnique literature and the VIII International Conference in Psychotechnique that took place in Prague in 1935.

psychotechniques / Czechoslovakia

Correo electrónico: hoskovec@mail.cz

* Traducción del inglés de Ramón León autorizada por Jiří Hoskovec. 
La psicotécnica es un área de la psicología aplicada relativamente joven. En el contexto internacional podemos recordar que Hugo Münsterberg, entonces profesor de la Universidad de Harvard, dio sus primeras conferencias sobre psicotécnica en la Universidad de Berlín en 1910. Su Psychologie und Wirtschaftsleben (Psicología y Economía), el primer libro que abordó de manera sistemática este tema, apareció en 1912.

Durante la Primera Guerra Mundial la psicotécnica se desarrolló rápidamente en Alemania y Estados Unidos. En Alemania los exámenes psicotécnicos estuvieron enfocados en la selección de pilotos y choferes, mientras que en Estados Unidos se desarrollaron tests de evaluación grupal de la inteligencia ampliamente utilizados en las fuerzas armadas.

En Checoslovaquia, el Instituto Psicotécnico (Psychotechnicky ústav) fue fundado en 1920 como el primero de los institutos establecidos dentro del marco de trabajo de la entonces recientemente establecida Academia Masarik del Trabajo (MAP).

La misión del instituto fue definida como la investigación acerca de la eficiencia del trabajo físico y mental y las aplicaciones de los hallazgos de dicha investigación (Brožek \& Hoskovec, 1986a; 1986b).

Las tareas del Instituto Psicotécnico fueron especificadas con gran detalle en un documento titulado "Organiza- ción y reglas básicas" (MAP PtÚ XIII.2.1141) y formuladas en 1926 como sigue: "Llevar a cabo investigación y actividades prácticas en el terreno de la psicotécnica (psychotechnika)".

Esto comprendía el análisis ocupacional, el desarrollo de métodos de la evaluación de las habilidades para actividades ocupacionales específicas, y el análisis estadístico de los resultados (con referencia a las intercorrelaciones de los puntajes de los test y su validación en términos de criterios de efectividad ocupacional.

Se destacó además que "los intereses del Instituto Psicotécnico se extenderán a todas las áreas del trabajo humano, incluyendo la industria, la empresa, el transporte, así como propósitos intelectuales".

De acuerdo con el rango cada vez mayor de responsabilidades, el título del Instituto cambió entre las dos guerras mundiales, de Instituto Psicotécnico a Instituto Psicotécnico Central (1932), y luego a Instituto del Trabajo Humano (1938).

Algunas comunicaciones nuestras previas tuvieron como propósito ofrecer contribuciones a la historia regional de la psicología en la hoy desaparecida Checoslovaquia. En el presente reporte consideraremos el desarrollo en un contexto más amplio, como una parte de la historia internacional de la psicología aplicada.

Trataremos estos temas en términos de las siguientes categorías de criterio: 
contactos personales, bidireccionales en su naturaleza, esto es, abarcando visitas y otros contactos profesionales de científicos y personal técnico del exterior, y visitas y largos periodos de estudio de psicotécnicos checos en otros países europeos. Consideraremos asimismo el intercambio de cartas, y las citaciones de autores extranjeros en publicaciones selectas y en congresos internacionales.

Los materiales pertinentes se encuentran en los Archivos Centrales de la Academia Checoslovaca de Ciencias, en un fólder designado MAP 361 XIII.8.1149, que lleva por título "Instituto Psicotécnico. Contactos con el extranjero. 1921-1926".

\section{CONTACTOS DIRECTOS}

El primer director del Instituto Psicotécnico, Petr Ružek, se formó como ingeniero y sirvió como asistente en la Universidad Técnica Checa en Praga. Él estuvo de acuerdo con el plan, aprobado por el consejo científico de la Academia Masaryk del Trabajo, de que un psicotécnico profesionalmente entrenado tuviera a su cargo los aspectos profesionales concernientes al Instituto. Desafortunadamente, en aquel momento no había alguien que tuviera ese perfil en Praga. Sobre la base de la recomendación del profesor William Stern, de Hamburgo, y del profesor Otto Lipmann, se hicieron arreglos con el doctor Walter Blumenfeld, de la Uni- versidad Técnica de Dresde, quien sirvió como consultor entre 1921 y 1922 y se trasladó luego de esa ciudad alemana a Praga.

\section{VISITAS Y ESTUDIOS EN EL EXTRANJERO}

Las visitas al extranjero llevadas a cabo por el personal del Instituto Psicotécnico fueron de dos clases: breves y largas. Por periodos cortos los psicólogos de Praga visitaron una serie de institutos en el extranjero, especialmente en Alemania.

E1 12 de mayo de 1922, el Instituto Psicotécnico de Praga formuló una solicitud a la Oficina Vienesa de Orientación Profesional Gratuita (Wiener Büro für unentgeltliche Berufsberatung) para permitir que dos profesionales visitaran la oficina para familiarizarse con los procedimientos que allí se desarrollaban.

Una comunicación enviada por el director del Instituto de Praga a la Embajada Checoslovaca en Viena, de fecha 29 de mayo de 1922, indica que se solicitó un permiso para visitar otra institución en la capital austriaca, la Hauptstelle der niederösterreichischen Landesberufsvormundschaft.

En setiembre de 1926, el ingeniero A. Dratva, de la división de construcción del Ministerio de Obras Públicas, y muy activo en la Academia Masaryk de Trabajo, visitó la Psychotechnische Versuchs-Stelle der Deutschen Reichsbahnen y la Verwaltungsakademie 
nebst Deutsches Institut für wirtschaftliche Arbeit in der öffentlichen Verwaltung.

Visitas de estudio más largas fueron llevadas a cabo por V. Forster a París en 1922 y 1923. Los viajes realizados a comienzos de los años 1920 por J. Stavel y por J. Váňa, incluyeron estancias de varios meses en Inglaterra, pero no están documentados en los materiales de archivo a los cuales hemos tenido acceso (cf. Váňa, 1926).

\section{VISITAS DEL EXTRANJERO}

En los años iniciales del Instituto Psicotécnico llegaron visitantes de Estados Unidos, Francia, Suiza, Alemania y España.

\section{Estados Unidos}

Un gran interés despertó la visita de Frank Gilbreth a Praga entre el 24 y el 28 de febrero de 1928. Dictó su conferencia el 25 de febrero en el Salón de Física de la Universidad Técnica Checa. El auditorio estuvo totalmente lleno. La presentación, acompañada por diapositivas, se efectuó en inglés con traducción simultánea al checo, siendo seguida con mucho interés. La ronda de preguntas y discusión se extendió por casi tres horas.

Gilbreth se concentró en los procedimientos de incremento de la productividad y el aumento de las ganancias por la reducción de costos. Anotó que algunas traducciones al alemán de sus escritos eran incorrectas y habían dado lugar a una presentación deformada de sus ideas. Sus actitudes hacia los métodos tradicionales de producción fueron críticas. Literalmente decía: los instrumentos y los equipos, tales como las sillas, son usualmente hechos como los féretros. El trabajador que hace un féretro no está en absoluto interesado en hacer uso de él. La persona que ordena un féretro y paga por él no lo necesita, mientras que aquel que va a hacer uso de él no puede lamentablemente decidir nada con respecto a la preparación de este.

La valoración de Gilbreth en las altas esferas se refleja en el hecho de que el 27 de febrero fue recibido en larga audiencia por T. G. Masaryk, presidente de Checoslovaquia, en su residencia de campo en Lány, cerca de Praga.

El reporte de "la visita del destacado ingeniero americano, preparado para los registros internos del Instituto Psicotécnico" (ítem 441/22) anota que "[...] él (F. G.) fue recibido muy cálidamente por el presidente y que consideró este encuentro como un gran evento en su vida".

\section{Francia y la Suiza de habla francesa}

Similarmente, la visita de I. Meyerson, director del laboratorio de psicología fisiológica en la Sorbona, atrajo gran atención. El 4 de octubre de 1923 Meyerson habló en la Sala del Instituto de Química de la Universidad Técnica 
Checa sobre la evaluación de la capacidad de rendimiento de los aviadores militares y civiles desde el punto de vista médico y psicotécnico. Usando diapositivas demostró algunos de los instrumentos empleados.

Esta conferencia fue considerada una excelente introducción en el uso de los métodos psicotécnicos para la utilización efectiva del factor humano.

Por recomendación de Pierre Bovet, director del Institut Jean Jacques Rousseau, de Ginebra, Martha Gairing (de Saint Gallen, Suiza) recibió una beca del Ministerio Checoslovaco de Educación, que hizo posible que observara y tomara parte en las actividades del Instituto Psicotécnico de Praga entre el 10 de setiembre y el 19 de octubre de 1925 .

\section{Alemania}

Una referencia previa fue hecha ya al contrato con el profesor Walter Blumenfeld de la Universidad Técnica de Dresde, para que proporcionara servicios de consultoría al Instituto Psicotécnico de Praga durante sus primeros dos años.

En 1928, el Instituto fue visitado por Jan Doležal, doctorado en la Universidad de Leipzig (en 1930), entonces asistente del Instituto Psicotécnico de la Universidad Técnica de Dresde. Doležal discutió la potencial participación del Instituto Psicotécnico de Praga en una nueva revista dedicada a la psi- cología aplicada, a ser editada por el profesor E. Sachsenberg, director del instituto de Dresde.

Subsecuentemente, el 7 de febrero de 1931, el profesor Sachsenberg efectuó una visita profesional al Instituto Psicotécnico de Praga, siendo acompañado por Jan Doležal, quien en 1932 se convirtió en vicedirector del instituto, rebautizado entre tanto como el Instituto Psicotécnico Central ( $c f$. Richter, 1981).

\section{España}

El psicotécnico español César de Madariaga sirvió como delegado de su país al Congreso Internacional de Management en Praga (abreviado como PIMCO), que se desarrolló en 1924. Aparentemente, hizo contactos personales con sus colegas checos.

En una carta fechada el 6 de febrero de 1926, el doctor Stangler, miembro de la II División del MAP, comunicó al Instituto Psicotécnico en Praga, que durante su reciente estancia en España tuvo la oportunidad de visitar también el Instituto de Reeducación Profesional del Trabajo, en Carabanchel Bajo, Madrid, donde De Madariaga le informó acerca de sus actividades en el instituto psicotécnico.

El doctor Stangler anota que está enviando una copia de una separata de un artículo de De Madariaga sobre psicotécnica, presentado en una conferencia en el Instituto de Ingenieros Civiles 
(De Madariaga, 1925), preservado en el Archivo Central de la Academia Checoslovaca de Ciencias (MAP 361 XIII.8).

Stangler sugiere que el equipo del Instituto Psicotécnico de Praga debería mantener contactos activos y colaboración con especialistas del exterior, incluyendo España, que, por varias razones, se encuentra aislada de otras naciones. Recomienda asimismo que De Madariaga sea nominado corresponsal extranjero ya sea del Instituto Psicotécnico o de la Academia Masaryk del Trabajo, en caso de que se creara ese tipo de membresía.

Se conservan en los archivos también varias fichas usadas por el laboratorio madrileño, tales como la ficha informativa, la psicológica, la psicotécnica y la fisiológica.

La ficha psicológica trata acerca de las funciones intelectuales (inteligencia general, atención, imaginación), varias clases de memoria (visual, auditiva), tiempos de reacción y sugestionabilidad.

La ficha "psicotécnica" sirve para registrar los resultados de la examinación de las funciones motoras (incluyendo fuerza, velocidad y precisión de los movimientos), fatigabilidad, y funciones perceptivas (percepción de formas, tamaño, peso, velocidades y ritmos).

\section{CORRESPONDENCIA}

Hemos dividido la correspondencia en dos categorías: 1) correspondencia pro- veniente del exterior; y, 2) correspondencia dirigida al exterior.

\section{Correspondencia proveniente del exterior}

P. Persant Snoep, un ingeniero holandés con un grado doctoral de la Universidad Técnica de Delft (disertación: Technisch-hygienische Beschowingen over de Economie van den Industriellen Arbeid, publicada en Leyden, Holanda), pregunta el 13 de enero de 1921 acerca de la posibilidad de familiarizarse con las actividades del Instituto Psicotécnico de Praga durante el verano de ese mismo año. Se le respondió indicándole que en setiembre sería bienvenido.

F. Paulsen, editor en jefe de la revista Bauwelt, en su comunicación del 29 de diciembre de 1921, informó acerca de observaciones iniciales referidas a los aspectos psicológicos y fisiológicos del colocado de ladrillos. Los materiales, obtenidos a pedido de la Forschungsgesellschaft für wirtschaftliches Baubetrieb, fueron depositados en el Architektenverein zu Berlín (Wilhelmstrasse 22).

En su nota de agradecimiento del 29 de marzo Frank Gilbreth, especialista norteamericano en management científico que visitaba Praga y daba conferencias, escribió: "Estoy colocando su nombre (doctor Ružek) entre los del Fatigue Committee of the Society of Industrial Engineers y estoy asimismo 
enviando en sobre aparte una copia de Nation's Health del 22 de febrero de 1922, que muestra el trabajo que uno de nuestros miembros, el profesor Henry J. Spooner, del Instituto Politécnico de Londres, está realizando. Su trabajo sobre eliminación de la fatiga está atrayendo una gran atención en ambos lados del Atlántico, y espero que usted pueda hallar su artículo de interés y valor". Gilbreth agrega: "Estoy también remitiendo aplicaciones para la membresía en la Taylor Society y en la Society of Industrial Engineers". En una comunicación posterior (carta del 13 de febrero de 1923), Gilbreth informa a Ružek que ha recibido las solicitudes de dos especialistas checos (C. J. Niederle y E. Slechta) y que las ha enviado a la American Society of Mechanical Engineers, junto con su aprobación.

El 17 de abril de 1922, Curt Piorkowski, director del Instituto de Estudio del Trabajo Humano y de la Psicotécnica (Institut für Arbeitswissenschaft und Psychotechnik, en Berlín), escribe una carta personal a Ružek, director del Instituto Psicotécnico de Praga, en la cual le informa que tiene trabajos no publicados sobre la medida y el amplio tema de la evaluación de las habilidades (Eignungsprüfungen). Vería con mucho agrado su publicación en checo y también en ruso, y escribe sobre el particular porque Ružek le habló acerca de sus planes de visitar Rusia.
El 6 de abril de 1922 Curt Piorkowski informa que la división psicotécnica de la Masaryk Academy de Trabajo ha conseguido todos los aparatos requeridos para evaluar las aptitudes de choferes de vehículos automovilísticos (tales como conductores de tranvías, choferes de charons e ingenieros de trenes).

Anota que el equipo es usado por la central de evaluación de la Streetcar Co. de Berlín y especifica los costos y arreglos para la entrega de los aparatos. Junto a ellos envía el reprint del Monatsblatter des Berliner Beziksvereins Deutscher Ingenieure, núm. 12 (diciembre de 1919, p. 159), de un artículo sobre "Eignungsprüfung und Ausbildung von Fahrzeugführern auf psychotechnischer Grundlage".

Cartas concernientes a aparatos psicotécnicos y sus costos fueron recibidas también de Hans Rupp (División de Psicología Aplicada, Instituto Psicológico de la Universidad de Berlín).

Una carta está fechada el 20 de agosto de 1912; la otra no tiene fecha. M. Pontégral (Comisariat Générale de la Republique Française), en una nota escrita en Estrasburgo el 3 de enero de 1923, acusa recibo de un cuestionario preparado por el Instituto Psicotécnico de Praga. Varias comunicaciones (25 de setiembre, 20 de noviembre, 3 de diciembre de 1923), tratan de las solicitud del doctor Forster para una membresía individual en la Personnel Research Federation (Nueva York). El en- 
cabezado de la carta especifica que el objeto de la federación entonces presidida por Robert M. Yerkes (Nacional Research Council) es "[...] la correlación de actividades de investigación pertinentes al personal en la industria, el comercio, la educación y el gobierno cuando sea que tales investigaciones son conducidas en el espíritu y con los métodos de la ciencia".

Un programa de cursos dado en el Institut de Psychologie de París incluye cursos de psicología aplicada, y demuestra que el Instituto de Praga estaba informado acerca de las actividades docentes en el exterior.

En una carta de fecha 19 de setiembre de 1925 y remitida desde Solothurn, Suiza, Franziska BaumgartenTramer, informa al doctor Forster que está escribiendo un libro sobre selección psicotécnica y que apreciaría recibir detallada información a acerca de los exámenes usados para evaluar a postulantes para ocupaciones tales como piloto de tranvía y conductores.

\section{Correspondencia dirigida al exterior}

En una carta del 10 de enero de 1921, Petr Ružek agradece a Aleš Hrdlička (curador de la División Antropológica del U. S. Nacional Museum, en el Smithsonian Institution, Washington, DC) por permitirle obtener "muestras de los exámenes psicotecnológicos". Ružek reconoce cálidamente el interés de Hrdlička en el instituto, entonces re- cién en sus comienzos, y anota que "la literatura especializada que está siendo publicada en América" sería muy bienvenida. Desde que se planea establecer varios centros de orientación vocacional, "libros y otras publicaciones en este campo" serían particularmente apreciados. Ružek también expresa interés por el intercambio de publicaciones futuras del Instituto Psicotécnico de Praga con instituciones similares en Estados Unidos. Las publicaciones del Instituto tendrían sumarios en inglés y francés.

Varias cartas fechadas el 10 de abril de 1923 se refieren a un reporte presentado en 1922 en un congreso de psicotécnica, en Milán, y enviadas para información (por ejemplo a Frank Gilbreth, en Estados Unidos, o al doctor G. van Wayenburg, en Amsterdam).

El 6 de junio de 1923, V. Forster, quien reemplaza a Ružek como director del Instituto de Praga, contacta por carta a Henri Piéron (Laboratorio de Psicología Fisiológica en La Sorbona), agradeciéndole por la amabilidad que se le demostró durante su estancia en París.

La visita a Praga que haría Piéron, con el apoyo del Ministerio Checoslovaco de Educación, fue considerada para el siguiente año académico (1923/1924). El consejo y la ayuda de Piéron serán muy apreciados. Concretamente, Forster le pide a Piéron confirmar si el equipo comprado a la firma Pirard y Boullet está en condiciones operativas. 
Se hace referencia también a la visita anticipada de I. Meyerson para dar conferencias públicas y consultoría con el staff del Instituto Psicotécnico.

El mismo día, 6 de junio de 1923, Forster escribe directamente a Meyerson (Laboratorio de Psicología Fisiológica de La Sorbona) para invitarlo a exponer en Praga con los auspicios de la Academia Masaryk del Trabajo. La respuesta de Meyerson no se encuentra en los expedientes pero la invitación del presidente de la Academia está fechada el 24 de agosto de 1923.

\section{ANÁLISIS DE CITAS}

Citas en la literatura y referencias en general proveen criterios objetivos para la cuantificación de la "realm of awareness".

Durante el periodo entre las dos guerras mundiales no existió ninguna revista dedicada específicamente a la psicología aplicada en Checoslovaquia. Los artículos relevantes de esta temática estuvieron muy diseminados.

Consecuentemente, hemos seleccionado tres publicaciones en forma de libros como fuentes de datos para el análisis de citas: 1) una introducción a la psicotecnología, publicada en los años veinte (Váňa, 1926); 2) una monografía sobre la medida de la inteligencia (Stejskal, 1934); y, 3) el primer volumen de la Encyclopedia of Efficiency (Verunáč, 1934).
Considerando que aquí estamos interesados en la interacción de los autores checos de los años 1920 y 1930 con el cuerpo de la literatura internacional, solo se tendrán en cuenta las referencias a trabajos publicados en otros idiomas diferentes del checo o el eslovaco. En los pocos casos en los cuales las traducciones son citadas, hemos acreditado la publicación al idioma en que apareció originalmente. Hubo problemas especiales en tratar con el trabajo de autores rusos citados en el texto de Stejskal (1934), que anota tres publicaciones en ruso y 16 publicaciones de autores rusos que aparecieron en alemán. Finalmente, hemos decidido tratar, no sin dificultad, todas las 19 publicaciones como "rusas". Las referencias en las actas de congresos internacionales fueron omitidas.

Tanto en Vana (1926) como en Verunáč (1934) las referencias son citadas en las bibliografías al final de los respectivos volúmenes. La bibliografía en Verunáč (1934) no es paralela a los capítulos del libro, sino que está clasificada por tópicos. Hemos analizado las referencias para 20 categorías consideradas como las más directamente relevantes, tales como la psicotécnica, la fatiga y la recuperación, y la consejería vocacional.

Los datos para cada una de las tres publicaciones que fueron examinadas y para las tres combinadas se presentan en la tabla 1. 
Tabla 1

Referencias a la literatura internacional en tres publicaciones checas

\begin{tabular}{lrcccc}
\hline & Inglés & Alemán & Francés & Ruso & Italiano \\
\hline Ván̆a $(1926, \mathrm{~N}=24)$ & 11 & 8 & 5 & & \\
Stejskal $(1934, \mathrm{~N}=341)$ & 132 & 144 & 46 & 19 & \\
Verunáč $(1934 ; \mathrm{N}=229)$ & 87 & 86 & 34 & 18 & 4 \\
\hline Total $(\mathbf{N}=\mathbf{5 9 4})$ & $\mathbf{2 3 0}$ & $\mathbf{2 3 8}$ & $\mathbf{8 5}$ & $\mathbf{3 7}$ & $\mathbf{4}$ \\
\hline
\end{tabular}

Las cifras indican una clara predominancia de las referencias en alemán e inglés. Habría sido deseable separar las referencias a los materiales publicados en Gran Bretaña y a los publicados en Estados Unidos. Desafortunadamente, tal separación no fue técnicamente posible. Los ecos de la literatura psicotécnica francesa son sustancialmente menores, y más asún las de autores rusos, y las referencias a los trabajos en italiano representan menos del $1 \%$ del total.

Sin embargo, uno puede ver los datos desde otra perspectiva, quizás más interesante. En términos de su conocimiento de la literatura técnica del día, los autores checos de las décadas de 1920 y 1930 estaban, en la práctica, orientados internacionalmente.

\section{CONGRESOS INTERNACIONALES}

El 29 de agosto de 1922, el director del Instituto Psicotécnico en Praga formula una pregunta en francés al Institut J. J. Rousseau, en Ginebra, Suiza, con la intención de averiguar si el instituto ginebrino y los psicólogos franceses par- ticiparían en el congreso internacional de Milán, y si los franceses habían admitido la participación de los alemanes.

La segunda parte de la comunicación trata un tema más amplio, o más precisamente, de dos temas: 1) el autor pregunta si el momento puede ser considerado como propicio para fundar bajo la égida de la International Labor Office, en Ginebra, un cuerpo internacional dedicado a intercambiar información con referencia a la elección vocacional y psicotécnica; y, 2) si en caso de que el grupo de Praga hiciera tal propuesta, sería recomendado y apoyado por el Institut J. J. Rousseau.

El profesor P. Bovet respondió el 23 de setiembre de 1923, recordando con placer su visita a Praga, incluyendo el Instituto Psicotécnico. Informó a su corresponsal que el Congreso Internacional de Psicotécnica estaba siendo organizado por el Institut J. J. Rousseau. Él (Bovet) no podrá estar presente, pero su colega. E. Claparède, director del Laoratorio de Psicología Experimental del Instituto, sí lo estará, como también 
Léon Walther, director de la Sección de Tecnopsicología del Instituto. A la carta de Bovet se agregó un volante (documento 496/22 P.U.), que describe un curso para consejeros psicológicos. Las conferencias tratarían tanto de orientación vocacional y gerencia científica (organización del trabajo, con secciones sobre antropometría, fisiología, psicología e higiene industrial). Los ejercicios prácticos comprenderían trabajo en el laboratorio, en plantas industriales, participación en la práctica de orientación vocacional y placement, y asistencia en consultas médicopedagógicas.

L. Walther, el spiritus movens de la tecnopsicología en el Institut J. J. Rousseau, planeó visitar el Instituto de Praga después del congreso de Milán. Bovet señaló que tanto los especialistas franceses como los alemanes asistirían al congreso de Milán, como ya lo habían hecho al de Barcelona. Él consideró buena la idea de Ružek con referencia al establecimiento de una sección de psicotécnica en conexión con el Bureau International de Travail y anotó que para el tiempo que vendría el Institut J. J. Rousseau proveería consultas a la International Labor Office en estas materias.

En una carta del 28 de junio de 1923 Léon Walther agradece a sus anfitriones de Praga por la cálida recepción en enero de ese mismo año y expresa su esperanza de que los contactos entre Ginebra y Praga se tornen más frecuen- tes. Walther anota que ha enviado, como material impreso, algunos test de aptitud empleados en el Institut J. J. Rousseau y él apreciaría su difusión entre los miembros del staff (J. Váňa y $K$. Lörsch), con quienes él habló de estas materias durante su visita.

Para la psicología aplicada checoslovaca el evento internacional más importante fue la VIII Conferencia Internacional de Psicotécnica, celebrada en Praga del 11 al 15 de setiembre de 1934 (Šeracky, 1935). Šeracky, profesor de psicología en la Universidad Carlos y director del Instituto Central de Psicotécnica, ejerció como presidente de este congreso.

La participación fue ampliamente internacional. Entre los conferenciantes españoles podemos anotar la presencia de A. Azoy (Barcelona), J. Germain (director del Instituto Nacional de Psicotécnica, de Madrid), Mercedes Rodrigo (Madrid) y J. Xirau (profesor de la Universidad de Barcelona). Germain, junto con C. de Madariaga (Madrid) y Emilio Mira y López (Barcelona) formaron parte del Comité Ejecutivo Internacional.

Numerosas presentaciones fueron hechas tanto por autores checos como alemanes. En la sección referida a la industria, los negocios y la administración, encontramos los reportes de A. Cibulka (prevención de accidentes en los steel mills en Vítvice) y E. Pechhold (selección de empleados en Vítkovice). En el área de transportes, J. Mls discutió 
el tema de la inteligencia con relación al rendimiento de los choferes de vehículos militares, mientras que J. Váňa reportó sobre accidentes de conductores de streetscar. En la sección de consejería, A. Smržová trató acerca de la selección de enfermeras, y J. Stavel abordó el psicodiagnóstico multidimensional.

Particular atención fue dada a la selección de estudiantes para la universidad (Doležal), la evaluación de los estudiantes universitarios (Matoušek) y exámenes psicotécnicos en las universidades, con especial énfasis en el análisis de los intereses de los estudiantes. K. Tríska discutió el problema de la velocidad como un factor en la capacidad de trabajo.

\section{INSTRUMENTOS PSICOLÓGICOS Y TEST}

En los años iniciales de la psicotécnica se prestó particular atención al uso de aparatos especializados. Algunos equipos fueron construidos en Checoslovaquia, como el aparato de reacción de elección múltiple de Forster, muy conocido en el ámbito internacional y ampliamente usado para la evaluación de pilotos y choferes de vehículos automovilísticos (Forster, 1928). Una modificación de ese aparato (llamado dispositivo Ostrava) es usada hasta la actualidad.

Los instrumentos usados en las evaluaciones psicotécnicas en general y en el Instituto Psicotécnico de Praga en particular están ilustrados en un capítulo escrito por Šeracky (1934).

La principal fuente de aparatos adquiridos por el Instituto Psicotécnico de Praga fueron Francia y Alemania. El equipo originario de Francia fue comprado con la ayuda del Ministerio de Defensa Nacional por recomendación de V. Forster, quien pasó algún tiempo en Francia en los años 1922 y 1923.

Los aparatos fueron comprados en Alemania a diferentes proveedores, incluyendo la famosa compañía de E. Zimmermann, en Leipzig. Un número de instrumentos provino de Industrielle Psychotechnik, localizada en Charlottenburg y dirigida por W. Moede. Debería anotarse que los miembros del staff participaron en cursos ofrecidos por los manufacturadores alemanes a sus usuarios, como el curso de tres meses que se dictó en Charlottenburg.

Deseamos anotar que un pequeño museo, adjunto al Departamento de Psicología, Universidad Carlos (dirección postal: Celetna 20,110 00 Praga 1), contiene una variedad de aparatos psicológicos construidos antes de la Segunda Guerra Mundial. Los aparatos sirven para la investigación en psicología experimental tanto como para las prácticas psicotécnicas. En adición, hay en el museo una colección de catálogos de tempranos aparatos psicológicos, sobre todo en alemán, algunos con descripciones en inglés, francés y español. Muchos aparatos están ilustrados en los catálogos (Hoskovec \& Štikar, 
1984). Unido al departamento está también un archivo de test de papel y lápiz y cuestionarios de diversas partes del mundo (Hoskovec \& RiČan, 1962 \& 1965).

\section{REFERENCIAS}

Brožek, J. \& Hoskovec, J. (1986a). K pocátkum uzite psychologie na Univerzite Karlove [Contribuciones a la historia temprana de la psicología aplicada en la Universidad Carlos, Praga). Acta Universitatis Carolinae. Historia Universitatis Carolinae Pragensis, 26, fasc. 1, 51-62.

Brožek, J. \& Hoskovec, J. (1986b). K ranémuinstitucionálnímu vyvoji uzité psychologie v Ceskoslovensku: Psychologie práce [Contribuciones al temprano desarrollo institucional de la psicología aplicada en Checoslovaquia: Psicología del trabajo humano]. Psychologie v ekonomické praxi, 21, 113-126.

Forster, V. (1928). A test for drivers. The Personnel Journal, 7, 3, 161-171.

Hoskovec, J. \& RiČan, P. (1962 \& 1965). Diagnosticke pomucky psychologicke.[Catálogo de los archivos de herramientas diagnósticas].
Praga: Psychologicky ustav, Zakladni katalog 1962, Dodatky 1965.

Hoskovec, J. \& Štikar, J. (1984). Historické pristroje $\mathrm{v}$ experimentální a pracovní Psychologii [Aparatos históricos en psicología experimental e industrial]. Psychologie $v$ economické praxi, 19, 2, 63-70.

Šeracky, Fr. (1934). Zprava o cinnosti UPUCsl. Za rok 1933 [Actividad del Instituto Psicotécnico Central (1933). En J. Dolezal \& Fr. Šeracky (Eds.). Psychotechnicka rocenka I. Praga: Orbis, 102-108.

Šeracky, Fr., (Ed.). (1935). Comptes rendus de la VIIIe conférence internationale de psychotechnique tenue a Prague du 11 au 15 septembre 1934. Praga: Orbis.

Stejskal, C. (1934). Detská inteligence [La inteligencia en el niño]. Praga: Profesorské nakladatelství.

Váňa, J. (1926). Industriální psychologie $\mathrm{v}$ Anglii [Psicología industrial en Inglaterra]. Nova Prace (Praga), 8, 263-265.

Verunac, V., (Ed.). (1934). Encyklopedie vykonnosti [Enciclopedia de eficiencia]. Sv. 1. Praga: Sfinx. 